\title{
Bacteriological profile and outcome of Ventilator associated pneumonia in Intensive care unit of a tertiary care centre
}

\author{
Ravi K', Maithili TM², David Mathew Thomas 3 , Sphoorti P Pai ${ }^{4}$ \\ ${ }^{1}$ Professor of Internal Medicine, ${ }^{3,4}$ Junior Resident, Department of Internal Medicine, Bangalore Medical College and \\ Research Institute (BMCRI), Bangalore, ${ }^{2}$ Consultant Physician, Metro Hospital, Shimoga, Karnataka, India
}

Background: Ventilator associated pneumonia (VAP) complicates the course of $8-28 \%$ of patients receiving mechanical ventilation. Appropriate antimicrobial treatment significantly improves the outcome. Hence rapid identification of infected patients and accurate selection of antimicrobials are important clinical goals. Aims and Objectives: The present study was conducted with an aim to know the outcome of VAP and to identify pathogens, compare the bacteriological profile, duration of mechanical ventilation and length of hospitalization. Materials and Methods: Sixty patients who developed VAP during our study period of 2 years were included after meeting inclusion and exclusion criteria. Study was conducted in Victoria hospital and Bowring \& Lady Curzon hospitals attached to Bangalore Medical College and Research institute. Results: Majority of patients were in the age group of 21-40 years. The occurrence of late VAP was $70 \%$. Klebsiella was the most common organism isolated in our study. Mortality was $13.3 \%$. Average duration of intubation was $13.1 \pm 6.6$ days. Duration of hospital stay was 16.2 \pm 7.1 days. Conclusion: Our study concluded that occurrence of late VAP was more common than early VAP. Targeted strategies aimed at preventing VAP should be implemented to improve patient outcome and length of hospitalisation. Above all utmost importance must be given to prevent VAP.

Key words: Intensive care unit, Ventilator associated pneumonia, Mechanical ventilator

\section{INTRODUCTION}

Nosocomial pneumonia is defined as an infection of the lung parenchyma that was neither present nor incubating at the time of hospital admission. Health-care associated pneumonias (HCAP) and nosocomial pneumonias are the second leading cause of hospital-acquired infections and are increasing in proportion due to the increased use of assisted ventilation and prolonged care of critically ill patients. Nosocomial pneumonias occur in $0.5-2.0 \%$ of hospitalized patients. ${ }^{1}$

A diagnosis of Ventilator associated Pneumonia (VAP) is arrived at, using clinical criteria when a patient who has been mechanically ventilated for $\geq 48$ hours develops a new or progressive infiltrate and the respiratory specimens are positive (i.e, increased neutrophils are seen in the microscopic analysis and growth of a pathogen in culture exceeds the set threshold). ${ }^{2}$

VAP is the second most common nosocomial infection, after urinary tract infection (UTI) and it has the highest fatality rate among nosocomial infections. ${ }^{3}$ The availability of accurate data on the epidemiology of VAP is limited by lack of standardised criteria for its diagnosis. In contrast to infections of more frequently involved organs like urinary tract and skin for which mortality is low, ranging from $1-4 \%$, the mortality rate for VAP ranges from $24-50 \%$ and can reach up to $76 \%$ in some settings. ${ }^{3}$

The pathogenesis of ventilator associated pneumonia is thought to be due to three principal mechanisms: 
(a) Introduction of bacterial flora into sterile lower respiratory tract (b) aspiration of pathogenic organism from upper respiratory tract or GIT (c) biofilm production on the endotracheal tube.

Risk factors for VAP include modifiable ones like supine position, gastric over-distension, colonisation of ventilator circuits, low pressure in endotracheal tube cuffs and reintubation. Non modifiable risk factors are male gender, age $>60$ years, history of COPD, presence of multi-organ dysfunction, cranial trauma and coma.

Early VAP is when the patient develops pneumonia in the first 4 days of mechanical ventilation. The most common organisms implicated are Moraxella catarrhalis, Hemophilusinfluenzae, Streptococcus pneumoniae. Late VAP develops after 5 days of ventilation. The spectrum of organisms changes to Gram negative pathogens like Acinetobacter baumanni, Pseudomonas aeruginosa, Klebsiella pneumonia. ${ }^{4,5}$

This study aims to know the outcome of VAP, to identify pathogens, bacteriological profile, duration of mechanical ventilation and length of hospitalization.

\section{MATERIALS AND METHODS}

The study was conducted over a period of 2 years extending from October 2012 to October 2014 in the ICU of Victoria hospital and Bowring \& Lady Curzon hospital attached to Bangalore Medical College \& Research Institute. A total of 60 patients of both sexes, age more than 18 years who were on mechanical ventilation for more than 48 hours were selected.

Clinical and diagnostic criteria were based on the Clinical Pulmonary Infection Score (CPIS). The CPIS originally proposed by Pugin and others helps in diagnosing VAP with a sensitivity of $72 \%$ and specificity of $80 \%$. It includes (1) new or progressive infiltrates in chest radiography (2) at least two of the following four (a) hypothermia or hyperthermia (b) presence of purulent secretions (c) leucocytosis or leukopenia (d) decreasing oxygen saturation.

Patients who developed pneumonia within 48 hours or those who were admitted with pneumonia and ARDS were excluded from the study. Age, sex, date of admission to ICU, date of initiating mechanical ventilation and mode of access to the patient airway were recorded. Indication of mechanical ventilation was noted. During initial stages of ventilation patients were adequately sedated. All necessary measures were taken for prevention of hospital acquired infections. Investigations done were complete blood count $(\mathrm{CBC})$, renal function tests (RFT), liver function tests (LFT), Chest X-ray, endotracheal aspirate (ETA) culture. ETA was obtained for microbiological quantitative cultures. A quantitative count of $10^{5} \mathrm{cfu} / \mathrm{ml}$ was labelled as VAP.

Once the diagnosis was established empirical antibiotic therapy was initiated and patient response was monitored.

\section{Statistical analysis}

Student t-test (two-tailed, independent) has been used to find out the significance of study parameters on continuous scale on metric parameters. Chi-square or Fischer Exact test has been used to find out the significance of the study parameters on categorical scale between two or more groups. The level of significance was set at $\mathrm{p}<0.05$. Statistical software: The Statistical software namely SPSS 15.0, Stata10.1, Med Calc 9.0.1 and R environment ver.2.11.1 were used for the analysis of the data and Microsoft word and Excel have been used to generate graphs, tables etc.

\section{RESULTS}

Sixty ICU patients aged 18 years or more, (min-max: 18-58years) who developed VAP were included in the study (Graph 1). Eighty one point seven percentage of the patients were males. A thorough clinical history, examination, investigations including chest radiographs, ET aspirate were obtained. The occurrence of late VAP $70 \%$ was more common than early VAP 30\% (Graph 2).

The study cohort comprised of patients of various cases of poisoning, neurological disorders, sepsis and others who developed VAP (Table 1). Main indication for ventilator support in patients who developed VAP was Organophosphorous poisoning (78.3\%).

Forty eight point three percent of the patients had bilateral consolidation, $33.3 \%$ patients had right lower zone consolidation on chest roentgenogram (Table 2).

Klebsiella was the most common organism isolated $(38.3 \%)$. Gram negative non fermenters in $18.3 \%$. Mixed infection was seen in $3(5 \%)$ cases (Tables 3 and 4$)$. Majority

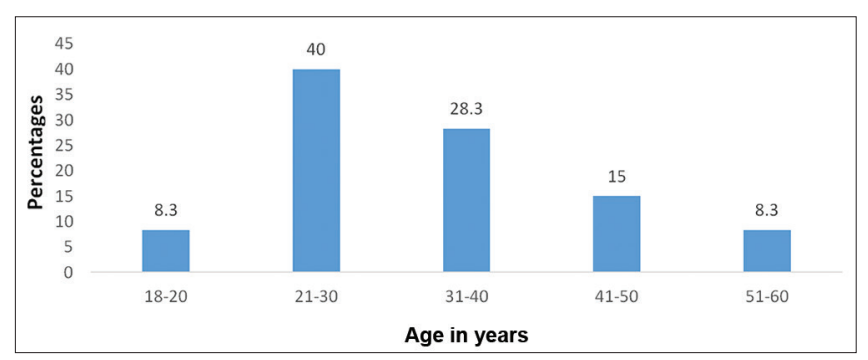

Graph 1: Age distribution 
of patients were treated with Piperacillin-tazobactam and Amikacin.

Most of the patients who developed VAP stayed in ICU between 8-14 days (Graph 3). Average duration of stay in ICU was 14.46 \pm 6.86 days and that in the hospital was $16.20 \pm 7.10$ days. Mortality occurred in 13.3\% and 85\% improved and were discharged home. Average duration of intubation was $13.08 \pm 6.60$ days.

Eight patients $(13.3 \%)$ died out of which 7 patients had late VAP and 1 patient had early VAP. 51(85\%) patients improved and were discharged and 1 patient left against medical advice (Graph 4). Comparison between early and late VAP was done (Table 5).

\section{DISCUSSION}

This study was done to know the outcome of patients in Ventilator Associated Pneumonia and to identify the pathogens causing VAP, how the development of VAP influenced duration of mechanical ventilation and length of hospitalization. Our study included 60 patients.

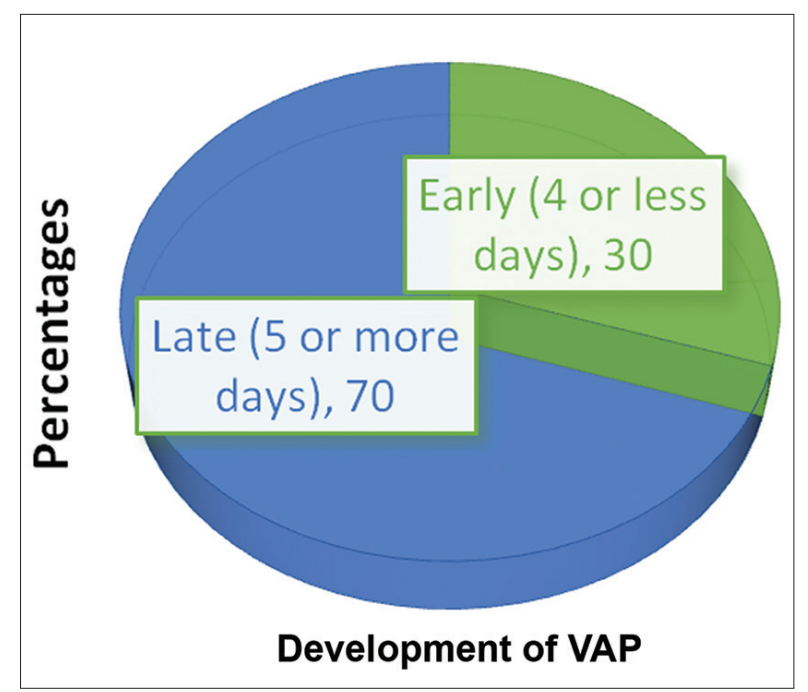

Graph 2: Development of VAP

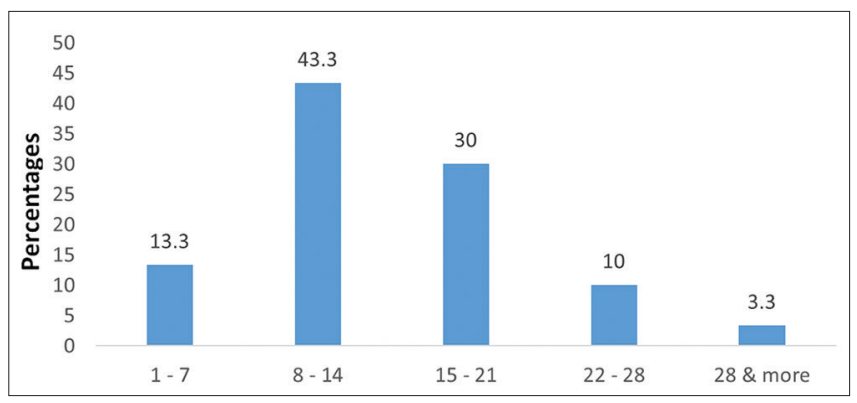

Graph 3: Duration of ICU stay in days
The incidence of VAP was more in males, it was statistically not significant. The mean age group in our study was 34 years. The young population group was attributed to the increased number of cases of poisoning that predominated our study. We divided our cases into early VAP ( $<96$ hours) and late VAP ( $>96$ hours). $42(70 \%)$ patients developed late VAP and $18(30 \%)$ patients developed early VAP.

Our study matches Gadani $\mathrm{H}$ et al as even his study included predominantly poisoning cases. In our study majority of cases who developed VAP were cases of organophosphorous poisoning. This could be because the poison causes excessive secretions and patient may get drowned in his own secretions.

\begin{tabular}{lc}
$\begin{array}{l}\text { Table 1: Diagnosis of patients studied } \\
\text { (alignment not proper) }\end{array}$ \\
\hline Diagnosis & $\begin{array}{c}\text { Number of } \\
\text { patients }(\mathbf{n}=60)(\%)\end{array}$ \\
\hline OPCP with respiratory failure & $47(78.3)$ \\
Acute demyelinating encephalomyelitis & $2(3.3)$ \\
Snake bite with neurotoxicity & $2(3.3)$ \\
Acute gastroenteritis & $1(1.7)$ \\
Bed bug poisoning & $1(1.7)$ \\
CVA-Intracerebral bleed & $1(1.7)$ \\
CVA posterior circulation stroke & $1(1.7)$ \\
CVT & $1(1.7)$ \\
Enterocutaneous fistula & $1(1.7)$ \\
Hanging & $1(1.7)$ \\
Meningitis & $1(1.7)$ \\
Unknown tablets consumption & $1(1.7)$ \\
\hline
\end{tabular}

\begin{tabular}{lc}
\hline Table 2: Repeat CXR after development of VAP \\
\hline Repeat CXR & Number of patients (\%) \\
\hline Bilateral consolidation & $29(48.3)$ \\
Left lower zone consolidation & $9(15.0)$ \\
Non homogeneous opacities all & $2(3.3)$ \\
over lung fields & \\
Right lower zone consolidation & $20(33.3)$ \\
Total & $60(100.0)$ \\
\hline
\end{tabular}

\begin{tabular}{lc} 
Table 3: Endotracheal tube culture \\
\hline Endotracheal tube culture & $\begin{array}{c}\text { Number of } \\
\text { patients (n=60) (\%) }\end{array}$ \\
\hline Klebsiella & $23(38.3)$ \\
Gram negative non fermenters & $11(18.3)$ \\
Gram negative fermenters & $1(1.7)$ \\
E.coli & $3(5.0)$ \\
Pseudomonas & $7(11.7)$ \\
Staphylococcus aureus & $7(11.7)$ \\
Coagulase negative staphylococcus & $4(6.7)$ \\
Gram negative non fermenters and & $1(1.7)$ \\
klebsiella & \\
Klebsiella and pseudomonas & $1(1.7)$ \\
MRSA & $1(1.7)$ \\
Pseudomonas and E.coli & $1(1.7)$ \\
\hline
\end{tabular}


Table 4: Correlation of pathogens associated according early and late VAP

\begin{tabular}{lccc} 
Pathogens & Early VAP $(\mathbf{n = 1 8 )} \mathbf{( \% )}$ & Late VAP $(\mathbf{n}=\mathbf{4 2})(\mathbf{\%})$ & $\mathbf{p}$-value \\
\hline Klebsiella & $5(27.8)$ & $18(42.9)$ & 0.387 \\
Gram negative non fermenters & $3(16.7)$ & $8(19)$ & 1.000 \\
Gram negative fermenters & $0(0)$ & $1(2.4)$ & 1.000 \\
E.coli & $2(11.1)$ & $1(2.4)$ & 0.576 \\
Pseudomonas & $3(16.7)$ & $4(9.5)$ & 0.419 \\
Staphylococcus aureus & $2(11.1)$ & $5(11.9)$ & 1.000 \\
Coagulase negative staphylococcus & $1(5.6)$ & $3(7.1)$ & 1.000 \\
Gram negative non fermenters\&klebsiella & $0(0)$ & $1(2.4)$ & 1.000 \\
Klebsiella and pseudomonas & $1(5.6)$ & $0(0)$ & 0.311 \\
MRSA & $0(0)$ & $1(2.4)$ & 1.000 \\
Pseudomonas and E.coli & $1(5.6)$ & $0(0)$ & 0.311 \\
\hline
\end{tabular}

\section{Table 5: Correlation of clinical and outcome variables according to early and late VAP}

\begin{tabular}{lcc} 
Variables & Early VAP $(\mathbf{n = 1 8 )}$ & Late VAP (n=42) \\
\hline Age & $34.27 \pm 11.16$ & $32.93 \pm 10.33$ \\
Males & $14(77.8 \%)$ & $35(83.3 \%)$ \\
Females & $4(22.2 \%)$ & $7(16.7 \%)$ \\
Duration of sensitive antibiotic & $5.05 \pm 0.99$ & 0.719 \\
Duration of ICU stay in days & $8.39 \pm 1.50$ & $6.02 \pm 0.99$ \\
Duration of hospital stay (post VAP) & $8.89 \pm 3.16$ & $17.07 \pm 6.60$ \\
Duration of intubation & $7.28 \pm 1.36$ & $12.69 \pm 7.30$ \\
Duration of stay in hospital & $9.94 \pm 1.95$ & $15.57 \pm 6.38$ \\
Death & $1(5.6 \%)$ & $0.001^{* *}$ \\
\hline
\end{tabular}

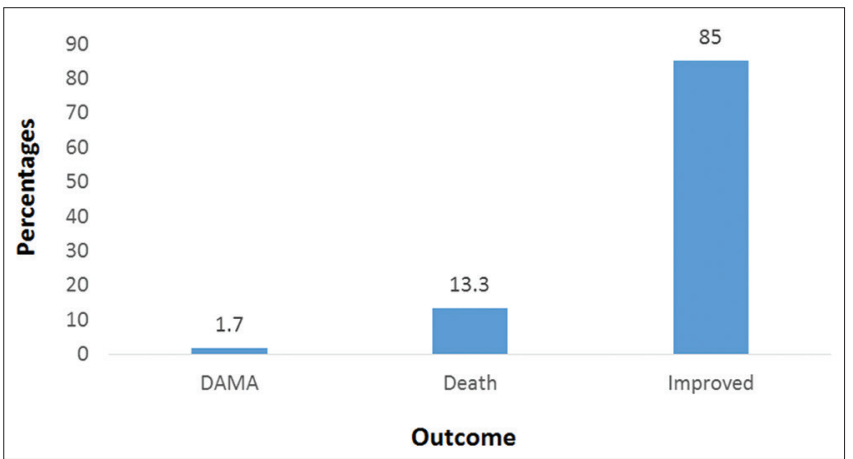

Graph 4: Outcome in patients with VAP

In our study the most common organism isolated was Klebsiella species which accounted for $38.3 \%$ of cases followed by gram negative non fermenters $(18.3 \%)$ and Pseudomonas (11.7\%). Organisms grown were similar in both early and late VAP in our study which was similar to study by Ibrahim et al. ${ }^{7}$ however in a study by Prod'hom et al it was seen that organisms in early VAP were different from those in late VAP. The organisms like Streptococcus pneumoniae, Hemophilus influenza and Methicillin sensitive Staphylococcus aureus were frequent cause of early VAP and organisms like Acinetobacter, Pseudomonas, Klebsiella species were encountered in late VAP.

The relationship between the duration of endotracheal intubation and development of VAP has been studied by several authors. Fagon et al. ${ }^{9}$ estimated an increased risk of $1 \%$ per day of mechanical ventilation. Torres et al. ${ }^{10}$ reported an increased incidence of VAP among patients ventilated more than 5 days compared to those with less than 5 days. Langer et al. ${ }^{11}$ demonstrated a high and constant rate of acquisition of VAP in first 8 to 10 days of endotracheal intubation with a lower rate thereafter. There is a similar trend of increasing lengths of endotracheal intubation and a high rate of acquisition of VAP in first 10 days of intubation in our study.

Patients who developed late VAP had longer duration of intubation with an average of $15.57 \pm 6.38$ days as compared to early VAP which was $7.28 \pm 1.36$ days. In a study conducted by Andales et al. ${ }^{12}$ the average duration of ventilation in late VAP was $12.67 \pm 8.10$ days and the length of hospitalisation was $23.40 \pm 12.93$ days. In our study the average length of hospitalisation in late VAP (18.88 \pm 6.82 days) was prolonged compared to early VAP (9.94 \pm 1.95 days). Eight patients (13.3\%) died out of which 7 patients had late VAP and 1 patient had early VAP.

\section{CONCLUSION}

We arrived at the following conclusions- Incidence of ventilator associated pneumonia is directly proportional to duration of mechanical ventilation and length of hospitalisation especially ICU stay. Klebsiella pneumonia was the most common organism isolated in our study. Mortality was higher among patients who developed late VAP. Simple and effective measure to prevent ventilator 
associated pneumonia should be employed in all ICU settings including staff education, hand hygiene, oral hygiene with chlorhexidine, nursing in semi recumbent position, promoting nasogastric feeds, performing closed suction of endotracheal tubes, minimising re-intubation and daily interruption of sedation.

The incidence of patients who are being admitted to ICU and requiring mechanical ventilation is increasing. Knowledge of causative microbial flora in a local setting would be vitally important to ensure more effective utilization of antibiotics and thereby, a better outcome. It would also allow formulation of strategies to decrease the incidence of VAP. There is an urgent need for many more hospital based prospective studies in our country.

\section{Limitations of the study}

Lack of a gold standard for diagnosis is the major culprit in poor outcome of VAP. Clinical diagnosis based on CPIS system poorly differentiates between VAP, pulmonary edema, pulmonary infarction and ARDS. Although microbiology helps in diagnosis it is not devoid of pitfalls. In fact it was proven that colonization of airway is common and presence of pathogens in tracheal secretions in the absence of clinical findings does not suggest VAP. In our study only endotracheal tube aspirate culture was done, no bronchoalveolar lavage was done.

\section{REFERENCES}

1. Bennani B, Selmani R, Mahmoud M, Nejjari C and Kanjaa N. Nosocomial pneumonia in mechanically ventilated patients: prospective study in intensive care unit of Fez university hospital. Saudi journal of Anaesthesia 20081; 2(2):46.

2. Kalil AC, Metersky ML, Klompas M, Muscedere J, Sweeney DA, Palmer LB, et al. Management of adults with hospital-acquired and ventilator-associated pneumonia: 2016 clinical practice guidelines by the Infectious Diseases Society of America and the American Thoracic Society. Clinical Infectious Diseases 2016; 63(5):e61-e111.

3. Apostolopoulou E, Bakakos P, Katostaras T and Gregorakos L. Incidence and risk factors for ventilator-associated pneumonia in 4 multidisciplinary intensive care units in Athens, Greece. Respiratory care 2003; 48(7):681-688.

4. Chastre $\mathrm{J}$ and Fagon JY. Ventilator-associated pneumonia. American journal of respiratory and critical care medicine 2002; 165(7):867-903.

5. Fagon JY, Chastre J, Hance AJ, Montravers P, Novara A and Gibert C. Nosocomial pneumonia in ventilated patients: a cohort study evaluating attributable mortality and hospital stay. The American journal of medicine 1993; 94(3):281-288.

6. Gadani H, Vyas A and Kar AK. A study of ventilator-associated pneumonia: Incidence, outcome, risk factors and measures to be taken for prevention. Indian journal of anaesthesia 2010; 54(6):535.

7. Ibrahim EH, Ward S, Sherman $\mathrm{G}$ and Kollef MH. A comparative analysis of patients with early-onset vs late-onset nosocomial pneumonia in the ICU setting. CHEST Journal 2000; 117(5):1434-1442.

8. Prod'hom G, Leuenberger P, Koerfer J, Blum A, Chiolero R, Schaller MD, et al. Nosocomial pneumonia in mechanically ventilated patients receiving antacid, ranitidine, or sucralfate as prophylaxis for stress ulcers randomized controlled trial. Annals of internal medicine 1994; 120(8):653-662.

9. Fagon JY, Chastre J, Domart Y, Trouillet JL, Pierre J, Darne C, et al. Nosocomial pneumonia in patients receiving continuous mechanical ventilation: prospective analysis of 52 episodes with use of a protected specimen brush and quantitative culture techniques. American Review of Respiratory Disease 1989; 139(4):877-884.

10. Torres A, Aznar R, Gatell JM, Jiménez P, González J, Ferrer A, et al. Incidence, risk, and prognosis factors of nosocomial pneumonia in mechanically ventilated patients. American Review of Respiratory Disease 1990; 142(3):523-528.

11. Langer $M$, Mosconi $P$, Cigada $M$ and Mandelli $M$. Long-term respiratory support and risk of pneumonia in critically III patients. American Review of Respiratory Disease 1989; 140(2):302-305.

12. Andales SA. The occurrence of late-onset ventilator associated pneumonia in the manila doctors hospital ICU: Risk Factors and clinical outcomes. The Philippine Journal of Microbiology and Infectious Disease 2004; 33(1):7-27.

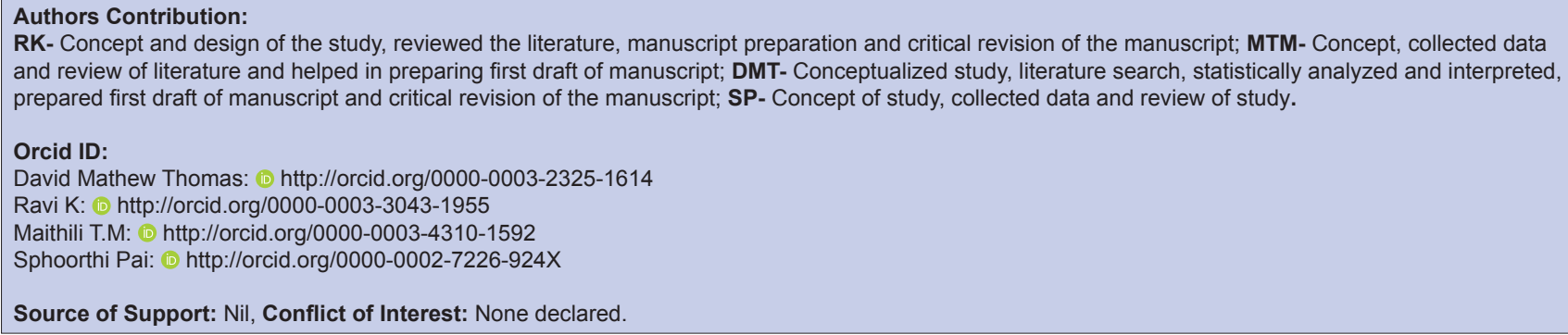

